medgen $2016 \cdot 28: 310-319$

DOI 10.1007/s11825-016-0102-6

Online publiziert:23. November 2016

(c) Der/die Autor(en) 2016. Dieser Artikel ist eine Open-Access-Publikation.

CrossMark

\author{
Joep Geraedts - Edith Coonen - Jos Dreesen - Aimee Paulussen - Christine de \\ Die-Smulders
}

Department of Clinical Genetics, Maastricht University Medical Centre+, Maastricht, Niederlande

\title{
Präimplantationsdiagnostik in den Niederlanden
}

rien dauerten 2 Jahre - blieben jedoch ohne Ergebnis. Erst 1995 wurde letztendlich PID auf einer experimentellen Basis zugelassen: PID wurde erlaubt, sofern ein hohes Risiko für genetisch bedingte, schwerwiegende Erkrankungen bestand, die nicht behandelt werden können. Nach Brüssel war Maastricht eines der ersten PID-Zentren in Zentraleuropa. 1997 wurde das erste holländische PIDBaby geboren. Eine Kostenerstattung durch das öffentliche Gesundheitswesen erfolgt seit 2006. Seitdem werden insgesamt 3 Behandlungszyklen pro Paar komplett erstattet. Sollte es während dieser Zyklen zu einer Schwangerschaft kommen, dann werden 3 weitere Zyklen ebenfalls erstattet.

Im Januar 2003 wurde gemäß Kap. 2 der Dutch Special Medical Procedures Act (Wet op de Bijzondere Medische Verrichtingen) durch das Ministry of Public Health, Welfare and Sports ein neues Zulassungssystem eingeführt. Zusätzlich zu den identischen Abrechnungszulassungen für die 8 holländischen klinischgenetischen Zentren wurde dem Maastricht University Medical Center (UMC) eine spezielle Erlaubnis erteilt, PID durchzuführen. Dies geschah vor dem Hintergrund der positiven Evaluation der in Maastricht implementierten PID. Die bestehenden Rahmenbedingungen änderten sich erst ab 2008, als die Kriterien für eine Zulässigkeit der PID durch die Ausweitung der Indikationen auf PID-Testung von BRCA1 und BRCA2 durch den stellvertretenden Minister of Public Health, Welfare and Sports erweitert werden sollten. Dieser Vorschlag wurde jedoch vom kleinsten Koalitionspartner blockiert und verursachte eine Regierungskrise. Kontrovers wurden insbesondere die unvollständige Penetranz, die variable Krankheitsexpression und die zur Verfügung stehenden therapeutischen Möglichkeiten bei erblichem Brust- und Eierstockkrebs diskutiert. Nach einer einmonatigen Diskussion und Verhandlung stimmte die überwiegende Mehrheit der holländischen Abgeordneten letztendlich für einen Kompromissvorschlag bezüglich des Testens von Embryonen auf genetisch bedingte Erkrankungen. In der Folge bestimmte die Regierung das University Medical Center (UMC) von Maastricht - das einzige universitäre Krankenhaus in den Niederlanden, das bisher bereits Embryoselektion durchführen durfte als Einrichtung, die die Erlaubnis für die Testung von Embryonen für eine größere Anzahl von Krankheitsentitäten erhielt. Der Kompromiss beinhaltete aber auch, dass die Regierung die Implementierung einer National Indications Commission durchsetzen konnte, die darüber bestimmen sollte, für welche neuen Krankheitsbilder eine PID in Frage kommen würde, vorausgesetzt, dass folgende Kriterien berücksichtigt werden würden:

1. die Schwere und Art der Erkrankung,

2. die Möglichkeiten der Prävention und Behandlung,

3. zusätzliche medizinische Kriterien und

4. psychologische und ethische Faktoren.

\section{Schwere und Art der Erkrankung}

Erforderlich ist ein hohes individuelles Risiko für eine schwerwiegende, genetisch bedingte Erkrankung. Die Schwere der Krankheit wird nicht definiert. 


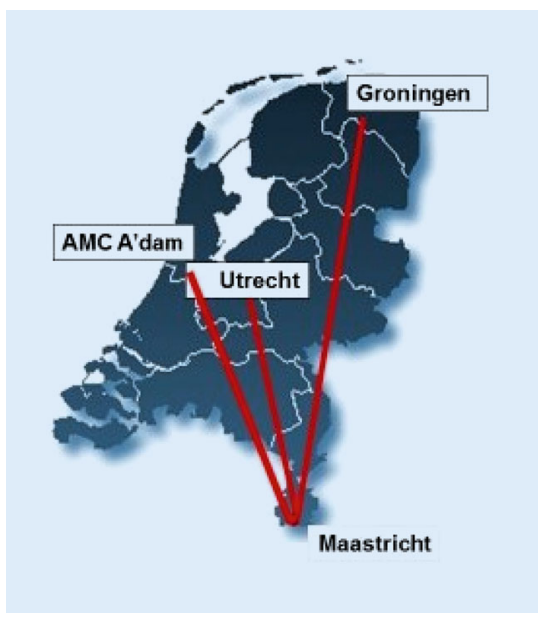

Abb. $1 \Delta$ Die Niederlande mit den PID-Zuweisungszentren

In einigen Fällen bleibt die Unterscheidung zwischen vollständiger und unvollständiger Penetranz unklar. Ein wichtiger Aspekt ist der Zeitpunkt des Krankheitsausbruches. Im Falle von hereditären Krebserkrankungen sollen folgende Aspekte berücksichtigt werden: Tumorrisiken für mehrere Organe, das Alter zum Zeitpunkt des Krankheitsausbruchs und die Schwere der Erkrankung in der betroffenen Familie.

\section{Die Möglichkeiten für Prävention und Behandlung}

Hierbei geht es um die Möglichkeiten der Diagnosestellung einer erblichen Erkrankung wie Krebs bereits im präklinischen Stadium der Erkrankung und einer Behandlung vor oder nach der klinischen Diagnosestellung. Um dies adäquat beurteilen zu können, sollen folgende Aspekte berücksichtigt werden: die Sensitivität und Spezifität der Testdiagnostik, die körperlichen Belastungen aufgrund der durchzuführenden Untersuchungen, die möglichen Risiken und Schäden, die im Zusammenhang mit einer (präventiven) Operation, Strahlen- und Chemotherapie zu erwarten sind, die Unfruchtbarkeit als Ergebnis einer Behandlung, die zu erwartenden Erfolge einer Behandlung und die Verfügbarkeit neuer Behandlungsformen.

\section{Zusätzliche medizinische Kriterien}

$\mathrm{Zu}$ den wichtigsten zusätzlichen medizinischen Kriterien zählen die Risiken einer IVF und einer Schwangerschaft für eine Frau, die Anlageträgerin für eine bestimmte genetisch bedingte Erkrankung ist (Beispiele hierzu vgl. auch Kapitel zu Methoden in diesem Artikel). Des Weiteren zählen hierzu mütterliches Alter, Unfruchtbarkeit und Übergewicht. Diese medizinischen Kriterien sollten auch bei der PID berücksichtigt werden, wie dies auch schon die Richtlinien für die IVF vorschreiben.

\section{Psychologische und ethische Faktoren}

Hauptfaktoren sind die psychologischen Belastungen. Weiterhin muss die Bereitschaft für unterschiedliche Reproduktionsmöglichkeiten (Pränataldiagnostik und Präimplantationsdiagnostik) sowie vorausgegangene Erfahrungen der Patientin (u. a. bspw. Schwangerschaftsabbruch/-abbrüche) berücksichtigt werden.

\section{Ausschluss einer Indikation für PID}

Geschlechtsbestimmung (aus sozialen Gründen) ist ebenso wenig zulässig wie dies in den meisten europäischen Ländern der Fall ist. Sogenannte Rettungskinderdiagnostik ist nur dann erlaubt, wenn eine PID aus genetischen Gründen indiziert ist. HLA-Typisierung ohne Indikation für eine PID aus genetischen Gründen ist nicht zulässig. Anlageträgerschaft als diagnostisches Kriterium ist nur dann erlaubt, wenn eine schwerwiegende genetische Erkrankung vorliegt, um diese beim Kind zu verhindern.

\section{Organisation der PID in den Niederlanden}

Die niederländischen PID-Richtlinien (Februar 2009) schreiben vor, dass in den Niederlanden nur ein Zentrum PID durchführen sollte. Diese Auflage widerspricht nicht der Möglichkeit, dass es mehrere Zentren gibt, die IVF anbieten, anschließend aber die Biopsien zur Analyse nach Maastricht schicken, wo sie den Anforderungen entsprechend bearbeitet werden. Die dafür notwendigen PIDTransfer-Dienstleistungen werden gegenwärtig von den IVF-Zentren Utrecht $U M C$, Groningen UMC und Amsterdam $A M C$ (• Abb. 1) angeboten.

Auf dem Hintergrund dieser Strukturen wird erreicht, dass in den Niederlanden das Angebot der PID auf 3 Ebenen organisiert ist: lokal, national und international.

\section{Lokal: Arbeitsgruppe PID Maastricht}

Seit 1995 trifft sich die lokale Arbeitsgruppe PID in Maastricht jeden Monat, um neue Fälle, Protokolle und die $\mathrm{Zu}$ sammenarbeit mit den in den Transfers eingebundenen Zentren etc. zu diskutieren. Sobald eine neue Krankheitsentität von der Arbeitsgruppe als Indikation für die PID akzeptiert worden ist, wird diese Indikation zur Genehmigung an die National Indications Commission eingereicht.

\section{National: PID Niederlande}

Das Maastricht University Medical Center (MUMC) hat mit den 3 universitären Zentren Embryonentransfervereinbarungen getroffen: mit dem UMC Utrecht seit 2008, mit dem UMC Groningen seit 2009 und mit dem Amsterdam Medical Center seit 2013. Diese Vorgehensweise schützt zukünftige Eltern aus anderen Teilen des Landes vor einer häufigen Anreise nach Maastricht, da ihre IVF regional durchgeführt und die Biopsie dann zur Diagnostik nach Maastricht weitergeleitet werden kann (•Abb. 1). Seit 2009 kooperieren das Diagnostikzentrum in Maastricht und die IVF Zentren unter den Namen PGD Nederland. Neue Entwicklungen, ethische Fragestellungen und organisatorische Angelegenheiten werden durch den sogenannten Vorstand diskutiert und behandelt, obwohl diese Organisation keinen eigenen rechtlichen Status hat. Rechtliche Angelegenheiten werden in bilateralen Verträgen zwischen der Maastricht UMC (als Lizenzhalter) und den universitären me- 
medgen 2016 28:310-319 DOI 10.1007/s11825-016-0102-6

(c) Der/die Autor(en) 2016. Dieser Artikel ist eine Open-Access-Publikation.

\section{J. Geraedts · E. Coonen · J. Dreesen · A. Paulussen - C. de Die-Smulders \\ Präimplantationsdiagnostik in den Niederlanden}

\section{Zusammenfassung}

Im Jahr 1995 wurde die Präimplantationsdiagnostik (PID) auf experimenteller Ebene in Maastricht eingeführt. Seit 2003 ist sie Bestandteil des Erstattungssystems des niederländischen Ministry Public Health, Welfare and Sport.

PID wird nur Paaren ermöglicht, die mit einem Risiko für eine schwerwiegende monogene Erkrankung, strukturellen Chromosomenanomalien oder mitochondrialen Erkrankungen bei ihren Nachkommen rechnen müssen. Ein Aneuploidiescreening zur Verbesserung der Erfolgsraten und assistierte Reproduktionstechnologien wie In-vitro-Fertilisation (IVF) oder Intrazytoplasmatische Spermieninjektion (ICSI) waren nie Gegenstand des PID-

Programms.

2008 beschloss die niederländische Regierung, eine "National Indications Commission" einzurichten, die bei neuen Krankheitsentitäten prüfen soll, ob die Kriterien für die Zulassung einer PID erfüllt sind: 1. die Schwere und Art der Erkrankung, 2. bestehende Möglichkeiten für Prävention und Behandlung, 3. zusätzliche medizinische Kriterien und 4. psychologische und ethische Faktoren.

Geschlechtsbestimmung (aus sozialen Gründen) ist auch in den Niederlanden, wie in den meisten europäischen Ländern, nicht erlaubt. Eine PID für die Diagnose sog. Rettungskinder ist nur dann erlaubt, wenn unabhängig hiervon eine Indikation für eine PID der genetischen Erkrankung besteht. HLA-Typisierung ohne Indikation für eine genetische Erkrankung ist hingegen nicht zulässig.

Das Maastricht University Medical Center (UMC) übernimmt die gesamte genetische Diagnostik und verfügt über die mit den Universitäten von Utrecht, Groningen und Amsterdam abgestimmten (University Medical Centre (UMC) Utrecht, University Medical Centre (UMC) Groningen and the
Amsterdam Medical Centre (AMC)) SOPs für die PID-Transporte.

Zwischen 1995 und 2015 wurden insgesamt 2870 Zyklen bei 1430 Paaren durchgeführt.

Der häufigste Grund für eine Überweisung zur PID war die Huntington-Krankheit, gefolgt von erblichem Brust- und Eierstockkrebs. Unter den weiteren Indikationen sind weit mehr autosomal-dominante genetisch bedingte Erkrankungen als autosomal-rezessive. Unter den zuletzt genannten stehen an erster Stelle Mukoviszidose und danach die spinale Muskelatrophie (SMA). Die Erfolgsrate liegt bei $20 \%$ pro Zyklusbeginn und bei $25 \%$ pro Embryonentransfer. Die Anzahl der Behandlungszyklen pro Paar liegt fast exakt bei 2,0 .

\section{Schlüsselwörter}

Präimplantationsdiagnostik · PID-Zuweisungszentren · Embryobiopsie · Einzelzelldiagnostik . Niederlande

\section{Preimplantation genetic diagnosis (PGD) in the Netherlands}

\section{Abstract}

In 1995, preimplantation genetic diagnosis (PGD) was introduced on an experimental basis in Maastricht. Since 2003 it has been part of the licencing system of the Ministry Public Health, Welfare and Sport.

PGD is only carried out for couples whose potential offspring are at risk of severe Mendelian disorders, structural chromosomal abnormalities or mitochondrial disorders. Preimplantation genetic screening, which is aneuploidy screening to improve medically assisted reproduction (IVF/ICSI) has never been part of the programme.

In 2008 the government decided to create a National Indications Commission, which determines for which new diseases PGD can be carried out using a number of criteria: 1. severity and type of disease; 2 . options for prevention and treatment; 3 . additional medical criteria; and 4. psychological and ethical factors.

Social sexing (family balancing for social reasons) is not allowed as is the case in most European countries. Saviour sibling diagnosis is only allowed in cases when PGD for a genetic disease is needed. HLA typing without PGD for a genetic disease is not allowed.

The Maastricht University Medical Centre does all genetic diagnoses and has protocollised contracts for transport PGD with the University Medical Centre Utrecht, University Medical Centre Groningen and the Amsterdam Medical Centre.

Between 1995 and 2015, a total of 2870

cycles were started in 1430 couples. The most frequent reason was Huntington disease followed by hereditary breast and ovary cancer. There are far more referrals and treatments for autosomal dominant diseases than for autosomal recessives. Among the latter, CF and SMA are the top indications. A total of 547 clinical pregnancies were obtained. The success of treatment is about $20 \%$ per started cycle and about $25 \%$ per embryo transfer. The number of treatments per couple is almost exactly 2.0 .

\section{Keywords}

Preimplantation genetic diagnosis - Transport PGD - Embryo biopsy - Single cell diagnosis . The Netherlands dizinischen Zentren, zu welchen die IVFLabore gehören, geregelt. Jedes Jahr im Herbst findet ein Treffen zwischen allen an der Durchführung der PID in den Niederlanden Beteiligten statt.

\section{PID International}

1995 startete die erste transnationale Zusammenarbeit mit dem PID-Zentrum der Freien Universität in Brüssel. Auf der Basis von bilateralen Verträgen wurde vereinbart, dass beide Zentren sich bei Problemfällen gegenseitig unterstützen. Des Weiteren findet jährlich das sogenannte BruMa-Meeting für all jene Fachgebiete statt, die an beiden Zentren an der Durchführung der PID beteiligt sind. Seit 2006 beteiligt sich das PID-Zentrum in Straßburg an der Zusammenarbeit und seit diesem Zeitpunkt finden die Treffen abwechselnd in Brüssel, Maastricht und Straßburg unter dem Namen BruMaStra statt. Jenseits des 
Tab. 1 Indikationen für alle Krankheitsbilder seit 2015

\begin{tabular}{|c|c|c|c|}
\hline & Gene & Paare & Zyklen \\
\hline Gesamt & & 360 & 500 \\
\hline PCR (gesamt) & & 266 & 372 \\
\hline Autosomal-dominante Krankheitsbilder & & 194 & 268 \\
\hline Erblicher Brust- und Eierstockkrebs & $B R C A 1 / 2$ & 45 & 60 \\
\hline Myotone Dystrophie Typ 1 (DM1) & $D M P K$ & 20 & 26 \\
\hline Huntington-Krankheit (direkter Test) & HTT & 21 & 26 \\
\hline Huntington-Krankheit (indirekter Test) & HTT & 12 & 14 \\
\hline Neurofibromatose Typ 1 (NF1) & NF1 & 15 & 23 \\
\hline Spinocerebelläre Ataxie Typ 3 (SCA3) & ATXN3 & 5 & 10 \\
\hline Familiäre Adenomatöse Polyposis (FAP) & $A P C$ & 9 & 9 \\
\hline Marfan-Syndrom & FBN1 & 6 & 9 \\
\hline Osteogenesis imperfecta Typ I & COL1A1 & 4 & 7 \\
\hline Hereditäre cerebrale Hämorrhagie (HCHWA-D) & $A P P$ & 4 & 6 \\
\hline Spastische Paraplegie Typ 4 (SPG4) & SPAST & 3 & 5 \\
\hline Erblicher Magenkrebs & $\mathrm{CDH} 1$ & 3 & 4 \\
\hline Erbliche motorische-sensible Neuropathie (HMSN1) & PMP22 & 2 & 4 \\
\hline Polyzystische Nierenkrankheit Typ 1 (AD PKD1) & PKD1 & 3 & 4 \\
\hline Tuberöse-Sklerose-Komplex 2 (TSC2) & TSC2 & 3 & 4 \\
\hline $\begin{array}{l}\text { Erbliches hereditäres nichtpolypöses kolorektales } \\
\text { Karzinom (HNPCC) }\end{array}$ & MLH1 & 1 & 3 \\
\hline Erbliche multiple Exostosen Typ 1 (HME1) & EXT1 & 2 & 3 \\
\hline Peutz-Jeghers-Syndrom & STK11 & 2 & 3 \\
\hline Hirschsprung-Krankheit & RET & 1 & 3 \\
\hline Amyotrophe Lateralsklerose (ALS)/FTD & C9ORF72 & 2 & 2 \\
\hline Hereditäre Leiomyomatose & $\mathrm{FH}$ & 1 & 2 \\
\hline Ektrodaktylie-Ektodermale-Dysplasie(EEC)-Syndrom & TP63 & 1 & 2 \\
\hline Erbliche multiple Exostosen Typ 2 (HME2) & EXT2 & 1 & 2 \\
\hline $\begin{array}{l}\text { Erbliches hereditäres nichtpolypöses kolorektales } \\
\text { Karzinom (HNPCC) }\end{array}$ & PMS2 & 2 & 2 \\
\hline Léri-Weill-Dyschondrosteose & SHOX & 1 & 2 \\
\hline Li-Fraumeni-Syndrom & TP53 & 1 & 2 \\
\hline Lipodystrophie Typ 2 (Dunnigan-Syndrom) & LMNA & 1 & 2 \\
\hline Noonan-Syndrom & PTPN11 & 1 & 2 \\
\hline Porencephalie und Peutz-Jeghers-Syndrom & $\begin{array}{l}\text { COL4A1+ } \\
\text { STK11 }\end{array}$ & 1 & 2 \\
\hline Retinoblastom und erblicher Brust- und Eierstockkrebs & $R B 1+B R C A 2$ & 1 & 2 \\
\hline Schwannomatose & SMARCB1 & 1 & 2 \\
\hline Von-Hippel-Lindau-Syndrom & $V H L$ & 1 & 2 \\
\hline $\begin{array}{l}\text { Warzen-Hypogammaglobulinämie-Immundefizienz- } \\
\text { Myelokathexis-(WHIM)-Syndrom }\end{array}$ & CXCR4 & 1 & 2 \\
\hline Aniridie & PAX6 & 1 & 1 \\
\hline Branchio-oto-renales Syndrom (BOR) & EYA1 & 1 & 1 \\
\hline CADASIL-Syndrom & NOTCH3 & 1 & 1 \\
\hline Kardiomyopathie (Desminopathie) & $D E S$ & 1 & 1 \\
\hline Cerebrocostomandibular-Syndrom (CCM) & KRIT1 & 1 & 1 \\
\hline Dyskeratosis congenita & TERC1 & 1 & 1 \\
\hline Familiäre Amyloid-Polyneuropathie & TTR & 1 & 1 \\
\hline
\end{tabular}

Austausches von Erfahrungen aus Klinik und Forschung, Protokollen und Daten, gibt es auch eine beschränkte Anzahl von gemeinsamen Projekten und Publikationen [15]. Die PGD Nederland ist Mitglied des ESHRE PID-Konsortiums.

\section{National Indications Commission PGD}

Im Herbst 2009 wurde die National Indications Commission gegründet. Einer Vorgabe des Ministeriums für Gesundheit folgend, initiierte der holländische Berufsverband für klinische Genetik (VKGN) und der Berufsverband der Gynäkologen und Geburtshelfer (NVOG) diese Kommission und benannte Mitglieder. Zusätzlich zu den Vertretern dieser beiden Berufsverbände gehören auch Patientenvertreter und Ethiker dieser Kommission an. Die oben genannten Kriterien stellen die Grundlagen für seine Entscheidungen dar. Die Kommission beurteilt nicht Einzelfälle per se. Vielmehr steht jeder Fall stellvertretend für den Umgang mit dieser Kategorie von Krankheitsbildern.

\section{Klinische Anwendungen in den Niederlanden \\ Aufnahmeprozedur}

Die Mehrheit der Patienten wird von einem Facharzt für Humangenetik aus einem der 8 niederländischen klinischen Zentren überwiesen, d. h. von dort, wo die Patienten eingangs beraten worden sind. Die PID erfordert eine vorangegangene erste genetische Testung. Hierzu werden die zukünftigen Eltern, ihr bereits erkranktes Kind oder andere Familienmitglieder, die möglicherweise Anlageträger einer bestimmten Erkrankung sind, einbestellt, um ihre DNA testen zu lassen. Während des Erstgesprächs an der Maastricht UMC werden Sie darüber informiert, wer einen präliminären genetischen Test durchführen lassen sollte. Ist die Entscheidung für eine PID getroffen, wird ein Termin mit einem Gynäkologen an der Maastricht UMC, Utrecht UMC, Groningen OMC oder Amsterdam AMC in Abstimmung mit dem betreffenden Paar vereinbart. Ein Reproduktionsme- 
Tab. 1 Indikationen für alle Krankheitsbilder seit 2015 (Fortsetzung)

\begin{tabular}{|c|c|c|c|}
\hline & Gene & Paare & Zyklen \\
\hline Holoprosencephalie & SHH & 1 & 1 \\
\hline Zapfendystrophie rot & GUCY2D & 1 & 1 \\
\hline Myotone Dystrophie Typ $1+22 q 11$ del & DMPK & 1 & 1 \\
\hline $\begin{array}{l}\text { Neurofibromatose Typ } 1 \text { (NF1) + } \\
\text { erbliche multiple Exostosen Typ } 1 \text { (HME1) }\end{array}$ & $N F 1+E X T 1$ & 1 & 1 \\
\hline Noonan-Syndrom & NRAS & 1 & 1 \\
\hline Paragangliom & $S D H B$ & 1 & 1 \\
\hline Retinoblastom + erblicher Brust- und Eierstockkrebs & RB1 & 1 & 1 \\
\hline Spastischen Paraplegie Typ 3A (SPG3A) & ATL1 & 1 & 1 \\
\hline Spinocerebelläre Ataxie Typ 17 (SCA17) & TBP & 1 & 1 \\
\hline Tuberöse-Sklerose-Komplex 1 (TSC1) & TSC1 & 1 & 1 \\
\hline Autosomal-rezessive Krankheiten & & 45 & 65 \\
\hline Spinale Muskelatrophie Typ 1/2 (SMA 1/2) & SMN1 & 11 & 16 \\
\hline Zystische Fibrose (CF) & CFTR & 10 & 15 \\
\hline Phenylketonurie & PAH & 1 & 3 \\
\hline Congenitale Taubheit & GJB2 & 2 & 2 \\
\hline Ehlers-Danlos-Syndrom Typ VIB & CHST14 & 1 & 2 \\
\hline Ellis-van-Creveld-Syndrom & EVC & 1 & 2 \\
\hline Gangliosidose Typ I & GLB1 & 1 & 2 \\
\hline Muskel-Auge-Gehirn-Krankheit (MEB) & POMGnT1 & 1 & 2 \\
\hline Nichtketotische Hyperglycinämie (NKH) & GLDC & 1 & 2 \\
\hline Osteogenesis imperfecta Typ I & CRTAP & 1 & 2 \\
\hline Rhabdomyolyse & LPIN1 & 1 & 2 \\
\hline Sichelzellenanämie/ $\beta$-Thalassämie & $H B B$ & 2 & 2 \\
\hline Tay-Sachs-Syndrom & HEXA & 1 & 2 \\
\hline Merosin-defiziente Muskeldystrophie & LAMA2 & 1 & 1 \\
\hline Morbus Gaucher & $G B A$ & 1 & 1 \\
\hline Dilatative Kardiomyopathie (DCM) & MYL2 & 1 & 1 \\
\hline Marinesco-Sjögren-Syndrom & SIL1 & 1 & 1 \\
\hline Metachromatische Leukodystrophie & ARSA & 1 & 1 \\
\hline $\begin{array}{l}\text { Mikrozephaler osteodysplastischer primordialer } \\
\text { Kleinwuchs Typ II (MOPD II) }\end{array}$ & PCNT & 1 & 1 \\
\hline Peters-Plus-Syndrom & B3GALTL & 1 & 1 \\
\hline Pontocerebelläre Hypoplasie Typ 2A & TSEN54 & 1 & 1 \\
\hline Sensenbrenner-Syndrom & IFT43 & 1 & 1 \\
\hline VICI-Syndrom & EPG5 & 1 & 1 \\
\hline Morbus Wilson & ATP7B & 1 & 1 \\
\hline X-gebunden (Mutationsanalyse) & & 25 & 37 \\
\hline Fragiles-X-Syndrom & FMR1 & 8 & 11 \\
\hline Muskeldystrophie Duchenne und Becker & $D M D$ & 5 & 7 \\
\hline Retinitis pigmentosa & $R P G R$ & 2 & 5 \\
\hline Incontinentia pigmenti (Bloch-Sulzberger-Syndrom) & NEMO & 2 & 4 \\
\hline Hämophilie A & F8 & 2 & 3 \\
\hline Adrenoleukodystrophie (ALD) & $A B C D 1$ & 1 & 2 \\
\hline Alport-Syndrom & COL4A5 & 1 & 1 \\
\hline Hämophilie B & F9 & 1 & 1 \\
\hline $\begin{array}{l}\text { Lujan-Fryns-Syndrom }+ \\
\text { erblicher Brust- und Eierstockkrebs }\end{array}$ & $\begin{array}{l}\text { MED12+ } \\
\text { BRCA2 }\end{array}$ & 1 & 1 \\
\hline
\end{tabular}

diziner wird dann entscheiden, ob beide Partner IVF-Kandidaten sind, indem er bei Ihnen eine Anamnese erhebt und sogenannte Fertilityparameter testet. Die Frau wird sich einer körperlichen Untersuchung unterziehen und es wird ein Ultraschall von den Eierstöcken vorgenommen. Bei dem Mann wird eine Samenanalyse durchgeführt. Beide, Mann und Frau, werden auf unterschiedliche Infektionserkrankungen hin untersucht.

\section{Methodische Aspekte}

\section{In-vitro-Fertilisation und -Kultur}

Die PID erfolgt vor einer künstlichen Befruchtung, die mit einer kontrollierten Ovarienstimulation beginnt, um möglichst qualitative hochwertige Oozyten zu gewinnen. Für den Fall, dass molekulare Amplifikationsmethoden genutzt werden, ist intrazytoplasmatische Spermieninjektion (ICSI) indiziert, um eine Kontamination der Spermien nach konventioneller IVF $\mathrm{zu}$ vermeiden. Bis vor kurzem wurden alle Biopsien an Tag 3 durchgeführt. Die Anzahl der Zellen am Tag der Entnahme ist variabel, aber die besten Embryonen bestehen zu diesem Zeitpunkt aus 8 oder mehr Zellen. In den meisten Fällen wird nur eine Zelle entnommen. Um die Menge des diagnostischen Materials einerseits zu erhöhen und andererseits weniger Mosaike $\mathrm{zu}$ untersuchen, wurde vor kurzem die Methode geändert und die Zellen erst am Tag 5 oder 6 während des Blastozystenstadiums des Embryos entnommen. Dies ermöglicht die Gewinnung mehrerer Zellen: In dieser Phase nach Befruchtung ist es möglich, ca. 5 Zellen dem Trophoblasten zu entnehmen. Nach der Trophoektodermbiopsie wird der Embryo so lange kryokonserviert bis er nach der Analyse zu einem späteren Zeitpunkt (im Laufe eines späteren Zyklus) rückübertragen werden kann.

\section{Genetische Diagnostik}

Im Prinzip können alle monogenen Erkrankungen, die mittels einer post- oder pränatalen Diagnostik analysiert werden können, auch im Rahmen einer PID untersucht werden. Bis vor kurzem wur- 


\begin{tabular}{|c|c|c|c|}
\hline & Gene & Paare & Zyklen \\
\hline Lymphangioproliferative Krankheit & $S H 2 D 1 A$ & 1 & 1 \\
\hline Pelizaeus-Merzbacher-Syndrom & PLP1 & 1 & 1 \\
\hline Mitochondrial & & 2 & 2 \\
\hline Leigh-Syndrom (m.14487T>C) & MTND6 & 1 & 1 \\
\hline $\operatorname{MELAS}(\mathrm{m} .3243 \mathrm{~A}>\mathrm{G})$ & MTTL1 & 1 & 1 \\
\hline FISH (gesamt) & & 38 & 48 \\
\hline X-gebunden (Geschlechtsanalyse) & & 8 & 11 \\
\hline Hämophilie A und B & & 2 & 4 \\
\hline Muskeldystrophie Duchenne und Becker & & 3 & 3 \\
\hline Lymphangioproliferative Krankheit & & 1 & 2 \\
\hline Alport-Syndrom & & 1 & 1 \\
\hline Lesch-Nyhan-Syndrom & & 1 & 1 \\
\hline Strukturelle Chromosomenabberationen & & 30 & 37 \\
\hline Reziproke Translokation, weibliche Anlageträgerin & & 13 & 15 \\
\hline Reziproke Translokation, männliche Anlageträger & & 9 & 11 \\
\hline Robertson-Translokation, männliche Anlageträger & & 3 & 4 \\
\hline $\operatorname{del}(X)(p 22.31)(p 22.31)$ & & 1 & 2 \\
\hline Inversion & & 2 & 2 \\
\hline $\operatorname{mos} 46, X X, \operatorname{del}(22)(q 13)[5] / 46, X X[95]$ & & 1 & 2 \\
\hline Robertson-Translokation, weibliche Anlageträger & & 1 & 1 \\
\hline Array (gesamt) & & 56 & 80 \\
\hline Strukturelle Chromosomenabberationen & & 56 & 80 \\
\hline Robertson-Translokation, männliche Anlageträger & & 13 & 17 \\
\hline Reziproke Translokation, männliche Anlageträger & & 18 & 26 \\
\hline Reziproke Translokation, weibliche Anlageträger & & 15 & 25 \\
\hline Robertson-Translokation, weibliche Anlageträger & & 6 & 7 \\
\hline Inversion & & 3 & 4 \\
\hline $\begin{array}{l}\text { Robertson-Translokation und reziproke Translokation, } \\
\text { weibliche Anlageträger }\end{array}$ & & 1 & 1 \\
\hline
\end{tabular}

de für alle monogenen Erkrankungen als Analysemethode PCR verwendet. Für die zystische Fibrose wurde bspw. ein Multiplex-Marker-PCR-Protokoll entwickelt [3]. Diese indirekte Methode ermöglicht das Verfolgen von Vererbungsmustern. Dies hat zur Konsequenz, dass sich die Familienmitglieder einem genetischen Test unterziehen müssen, damit informative polymorphe Marker identifiziert werden können. Diese Information kann dann genutzt werden, um zu überprüfen, ob der Embryo den betroffenen Genotyp hat oder nicht. PID-PCR ist anfällig für die Kontamination mit extraembryona-
Das genomweite Karyomapping istextrem akkurat und erleichtert die Analyse von fast allen monogenen Erkrankungen bzw. jeder Kombination von Loci auf Einzelzellniveau. Sie ermöglicht damit eine Ausweitung des Indikationsgebietes für die PID, ohne dass vorher ein Routinetest entwickelt werden muss [9].

Viele Jahre hindurch wurde PID für Huntington-Chorea mittels direkter Testverfahren durchgeführt bzw. Vorhandensein oder Abwesenheit des mutierten Allels auf Basis der elterlichen Allele des Huntington-Disease(HD)Gens geprüft [13]. In jüngerer Zeit wurde ein Ausschlusstest zugelassen. Diese Strategie erlaubt nun die Identifikation der mütterlichen und väterlichen vererbten Allele und kann in der Folge dazu genutzt werden, die Niedrigrisikoallele beim Ungeborenen $\mathrm{zu}$ identifizieren. Jenseits eines geringen Risikos von Rekombinationsereignissen kann diese Methode dazu genutzt werden, um zu entscheiden, ob das Individuum ein niedriges oder hohes Risiko trägt, diese Krankheit zu entwickeln [8].

Weiterhin wurde in allen Protokollen Bezug darauf genommen, dass Frauen, die von bestimmten genetischen Erkrankungen betroffen sind, selbst ein erhöhtes Risiko tragen für Probleme, die in engem Zusammenhang stehen mit IVFBehandlung und/oder Schwangerschaft und/oder Geburtskomplikationen.

Komplikationen können in jeder Phase der IVF- oder PID-Behandlung entstehen und sind möglicherweise unterschiedlichen Ursprungs. In der Stimulierungsphase besteht insbesondere bei Anlageträgerinnen für das Fragile-XSyndrom ein erhöhtes Risiko für eine geringe Oozytenausbeute [7]. Ein erhöhtes Risiko für Blutungskomplikationen während oder nach der Oozytenentnahme besteht für Hämophilieanlageträgerinnen [10]. Frauen, die an einer genetisch bedingten Kollagenerkrankung leiden, besitzen möglicherweise ein erhöhtes Risiko für kardiovaskuläre Komplikationen [11].

Hinsichtlich der Analyse von Chromosomentranslokationen wurden unterschiedliche Vorgehensweisen auf der Basis von FISH entwickelt [12]. Erst vor kurzem wurde komparative genomische Hy- 
Tab. 2 Indikation aller durchgeführten PID-Behandlungen in den Niederlanden, 1995-2015

\begin{tabular}{|c|c|c|c|}
\hline Monogene Erkrankungen & Gene & Paare & Zyklen \\
\hline Gesamt & & 1430 & 2870 \\
\hline PCR (gesamt) & & 802 & 1735 \\
\hline Autosomal-dominante Krankheiten & & 532 & 1198 \\
\hline Huntington-Krankheit, direkter Test & HTT & 105 & 244 \\
\hline Huntington-Krankheit, indirekter Test & HTT & 17 & 40 \\
\hline Erblicher Brust- und Eierstockkrebs & $B R C A 1 / 2$ & 98 & 214 \\
\hline Myotone Dystrophie Typ 1 (DM1) & DMPK & 88 & 198 \\
\hline Familiäre adenomatöse Polyposis (FAP) & $A P C$ & 26 & 73 \\
\hline Marfan-Syndrom & FBN1 & 26 & 58 \\
\hline Neurofibromatose Typ 1 (NF1) & NF1 & 24 & 53 \\
\hline Spinocerebelläre Ataxie Typ 3 (SCA3) & ATXN3 & 19 & 49 \\
\hline Andere & & 129 & 269 \\
\hline \multicolumn{4}{|l|}{ Autosomal-rezessive Krankheiten } \\
\hline Zystische Fibrose (CF) & CFTR & 58 & 121 \\
\hline Spinale Muskelatrophie Typ 1 oder 2 (SMA1/2) & SMN1 & 38 & 74 \\
\hline Pontocerebelläre Hypoplasie Typ 2a (PCH2a) & TSEN54 & 4 & 9 \\
\hline Kongenitale Glykosylierungsstörung (CDG) 1a & PMM2 & 2 & 6 \\
\hline Beta-Thalassämie & $H B B$ & 3 & 5 \\
\hline Andere & & 66 & 124 \\
\hline X-gebunden (Mutationsanalyse) & & 88 & 179 \\
\hline Fragiles-X-Syndrom & FMR1 & 46 & 96 \\
\hline Muskeldystrophie Duchenne und Becker & $D M D$ & 12 & 28 \\
\hline Hämophilie A & F8 & 6 & 10 \\
\hline Andere & & 24 & 45 \\
\hline Mitochondrial (Mutationsanalyse) & & 11 & 19 \\
\hline MELAS m.3243 A>G & MT-TL1 & 7 & 12 \\
\hline Leigh/NARP m.8993 C>T/G & MT-ATP6 & 2 & 4 \\
\hline Leigh (m.14.487 T>C) & MT-ND6 & 1 & 2 \\
\hline MERRF $m .8344 \mathrm{~A}>\mathrm{G}$ & MT-TK & 1 & 1 \\
\hline FISH (gesamt) & & 530 & 995 \\
\hline X-gebunden (Geschlechtsanalyse) & & 133 & 271 \\
\hline Hämophilie A/B & & 33 & 64 \\
\hline Muskeldystrophie Duchenne und Becker & & 23 & 48 \\
\hline Androgenresistenz-Syndrom & & 6 & 10 \\
\hline Andere & & 71 & 149 \\
\hline Autosomal-dominant (Geschlechtsanalyse) & & 1 & 2 \\
\hline Erblicher Brust- und Eierstockkrebs (BRCA1) & & 1 & 2 \\
\hline Mitochondrial (Geschlechtsanalyse) & & 3 & 4 \\
\hline Hereditäre Leber-Optikusneuropathie (LHON) & & 3 & 4 \\
\hline
\end{tabular}

bridisierung $(\mathrm{CGH})$ eingeführt in Form einer Array-CGH zur Analyse von strukturellen Chromosomenanomalien.

\section{Ergebnisse}

- Tab. 1 gibt einen Überblick über alle Indikationen der 500 Behandlungen im Jahr 2015. Dabei überwog die Indikation für monogene Erkrankungen gegenüber strukturellen Chromosomenanomalien. In der Mehrheit der Fälle gab eine autosomal-dominante Erkrankung den Ausschlag, eine PID in Anspruch zu nehmen. In dieser Gruppe gehörte zu den Hauptindikationen erblicher Brustund Eierstockkrebs, gefolgt von Huntington-Krankheit und Myotoner Dystrophie Typ 1 (DM1). Bei mehr als zwei Drittel aller Fälle mit einer zugrunde liegenden Huntington-Krankheit wurde ein direkter Test gewählt. Insgesamt wurden mehr als 50 autosomal-dominante Erkrankungen diagnostiziert. Was die autosomal-rezessiven Krankheiten betrifft waren SMA und Mukoviszidose weitaus häufiger repräsentiert als alle anderen 22 Erkrankungen dieses Erbgangs.

$\mathrm{Zu}$ den häufigsten X-chromosomalen Krankheitsbildern zählte das Fragile-X-Syndrom und die Muskeldystrophie Duchenne/Becker. Eine FISH-Diagnostik zur Geschlechtsbestimmung im Falle von X-chromosomalen Krankheitsbildern wurde mehr und mehr obsolet. Des Weiteren wurde die FISH-Diagnostik ersetzt durch Array-CGH zur Diagnostik struktureller Chromosomenanomalien. Die häufigste Indikationsstellung in dieser Gruppe bezog sich auf die reziproke Translokation - beide Geschlechter waren als Anlageträger hier gleich repräsentiert. Bei den Robertson-Translokationen gab es jedoch eindeutig mehr männliche Anlageträger.

- Tab. 2 zeigt die Ergebnisse seit Beginn 1995 bis 2015: Insgesamt gab es 2870 Zyklen bei 1430 Paaren. Mehr oder weniger ergibt sich das gleiche Bild wie 2015, obwohl sich leichte Veränderungen ergeben haben: Insgesamt überwogen die Anfragen wegen der Huntington-Krankheit gegenüber erblichem Brust- und Eierstockkrebs. Die gleiche Beobachtung lässt sich für alle Anfragen aufgrund der Indikation für Mukoviszidose gegenüber 
Tab. 2 Indikation aller durchgeführten PID-Behandlungen in den Niederlanden, 1995-2015 (Fortsetzung)

\begin{tabular}{|c|c|c|c|}
\hline Monogene Erkrankungen & Gene & Paare & Zyklen \\
\hline Strukturelle Chromosomenabberationen & & 393 & 718 \\
\hline Reziproke Translokation & & 274 & 498 \\
\hline Robertson-Translokation & & 87 & 165 \\
\hline Deletion & & 14 & 29 \\
\hline Inversion & & 9 & 11 \\
\hline Insertion & & 4 & 6 \\
\hline $\begin{array}{l}\text { Robertson-Translokation } \\
\text { und reziproke Translokation }\end{array}$ & & 2 & 4 \\
\hline Wiederholt auftretende Trisomie 21 & & 1 & 2 \\
\hline Mosaik-Turner-Syndrom & & 2 & 2 \\
\hline Array (gesamt) & & 98 & 141 \\
\hline Strukturelle Chromosomenabberationen & & 98 & 141 \\
\hline Reziproke Translokation & & 54 & 79 \\
\hline Robertson-Translokation & & 35 & 46 \\
\hline Deletion & & 2 & 5 \\
\hline $\begin{array}{l}\text { Robertson-Translokation } \\
\text { und reziproke Translokation }\end{array}$ & & 3 & 6 \\
\hline Inversion & & 4 & 5 \\
\hline
\end{tabular}

Tab. 3 Gesamtanzahl der PID-Behandlungen von 1995 bis 2015 und Schwangerschaftsraten

\begin{tabular}{|l|r|r|r|r|} 
& Insgesamt & PCR & FISH & \multicolumn{1}{l}{ Array } \\
\hline Gestartete Paare & 1430 & 802 & 530 & 98 \\
\hline Gestartete Zyklen & 2870 & 1735 & 994 & 141 \\
\hline Kryokonservierte Embryonen vor Analyse & 54 & 37 & 14 & 4 \\
\hline Eizellpunktionen & 2437 & 1508 & 815 & 114 \\
\hline $\begin{array}{l}\text { Analysierte Zyklen } \\
\text { Kryokonservierte Embryonen nach Analyse }\end{array}$ & 2407 & 1489 & 808 & 110 \\
\hline Zyklen mit frischem Embryotransfer & 576 & 415 & 218 & 18 \\
\hline Zyklen mit Embryotransfer nach Auftauen & 1849 & 1218 & 544 & 87 \\
\hline $\begin{array}{l}\text { Klinische Schwangerschaften }>12 \text { Wochen nach } \\
\text { frischem Zyklus }\end{array}$ & 537 & 390 & 126 & 13 \\
\hline $\begin{array}{l}\text { Klinische Schwangerschaften }>12 \text { Wochen nach } \\
\text { Auftauen }\end{array}$ & 484 & 307 & 150 & 27 \\
\hline Klinische Schwangerschaften insgesamt & & & & 16 \\
\hline
\end{tabular}

Schwangerschaftsraten (\%) nach 12 Wochen pro:

\begin{tabular}{|l|l|l|l|l|}
\hline gestartetem Zyklus & 19,1 & 20,3 & 16,7 & 19,9 \\
\hline Eizellpunktion & 22,4 & 23,4 & 20,4 & 24,6 \\
\hline Embryotransfer & 22,9 & 22 & 24,8 & 28 \\
\hline Embryotransfer in frischem Zyklus & 26,2 & 25,2 & 27,6 & 31 \\
\hline Embryotransfer nach Auftauen & 11,7 & 11,8 & 12,7 & 7,7
\end{tabular}

der Indikation für eine SMA machen. Darüber hinaus gab es in der Vergangenheit etwas mehr Anfragen zur Geschlechtsbestimmung aus Gründen einer X-chromosomalen Krankheit.
Tab. 4 Anzahl der PID-Behandlungen pro

IVF-Zentrum

\begin{tabular}{|l|r|r|}
\hline & Paare & Zyklen \\
\hline Maastricht & 728 & 1556 \\
\hline Utrecht & 497 & 917 \\
\hline Groningen & 120 & 256 \\
\hline Amsterdam & 85 & 141 \\
\hline Gesamt & 1430 & 2870 \\
\hline
\end{tabular}

die Zahl der Behandlungen pro Paar fast exakt bei 2 liegt.

Wir hatten bisher nur eine Fehldiagnose: Dabei handelte es sich um den Fall einer sehr schwierig zu diagnostizierenden chromosomalen Translokation [14].

\section{Rückblick}

Zu Beginn der PID-Behandlung auf experimenteller Basis im Jahr 1995 wurde die PID nur dann zugelassen, wenn es sich um ein hohes Risiko für eine nichtbehandelbare schwere, genetisch bedingte Erkrankung handelte. Bei den sehr schweren Fällen mit frühem Krankheitsausbruch handelt es sich sehr häufig um autosomal-rezessive Erkrankungen. In den meisten Fällen bedeutete dies, dass es bereits ein betroffenes Kind in der Familie gab und die Eltern diese Erkrankung beim nächsten Kind vermeiden wollten. Schon bald nach Beginn wurden wir von den verschiedenen Patientenselbsthilfegruppen kontaktiert. Die Huntington Association gehörte hier zu den ersten. Wunschgemäß diskutierten wir die vorsichtige Einführung eines direkten Testangebots. Ein indirekter oder verdeckter Test wurde nicht angeboten.

Gegenwärtig sind autosomal-dominante Krankheitsbilder im Allgemeinen und die Huntington-Krankheit im Speziellen wesentlich häufigere Gründe für eine PID als autosomal-rezessive. Offensichtlich denken Ratsuchende/Patienten anders als die Kritiker, die behaupten, dass die Anwendung PID im Falle einer Huntington-Krankheit unverantwortlich sei, weil vor dem Kind noch viele symptomfreie Jahre liegen würden. Dies jedoch zollt nicht dem Bild der harten Realität Tribut: Diese Erkrankung ereilt Menschen in der Blüte ihres Lebens, nimmt einen fatalen Verlauf und wirft ihre langen Schatten voraus [4]. Mehr 
oder weniger dasselbe gilt für genetisch bedingte Erkrankungen mit unvollständiger Penetranz und präventiven/ therapeutischen Optionen, wie z. B. bei erblichen Tumoren. Nach langen Diskussionen hinsichtlich der Akzeptanz des PID-Einsatzes bei erblichem Brustund Ovarialkrebs sicherten wir der holländischen Regierung zu, jährlich einen Bericht mit allen Behandlungsdaten aus dem Vorjahr zu veröffentlichen. Seit 2009 ergeht dieser Jahresbericht an das holländische Parlament mit der Folge, dass viele Fragen aufgeworfen wurden. Diese konnten jedoch zufriedenstellend von unserer Seite beantwortet werden. Letztendlich waren unsere Behandlungsergebnisse mit jenen des ESHRE PIDKonsortiums vergleichbar [6].

\section{Korrespondenzadresse}

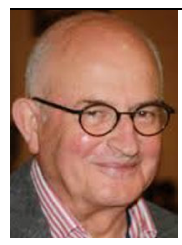

\section{J. Geraedts}

Department of Clinical Genetics, Maastricht University Medical Centre+ P.O. Box 5800, 6202 AZ Maastricht, Niederlande joep.geraedts@mumc.nl

Danksagung. Wir möchten uns bei allen Kolleginnen und Kollegen, die Mitglied der PGD Nederland sind, für ihre aktive Beteiligung bedanken.

Open access funding provided by Maastricht University Medical Center (UMC+).

\section{Einhaltung ethischer Richtlinien}

Interessenkonflikt. J. Geraedts, E. Coonen, J. Dreesen, A. Paulussen und C. de Die-Smulders geben an, dass kein Interessenkonflikt besteht.

Alle beschriebenen Untersuchungen am Menschen wurden mit Zustimmung der zuständigen EthikKommission, im Einklang mit nationalem Recht sowie gemäß der Deklaration von Helsinki von 1975 (in der aktuellen, überarbeitete Fassung) durchgeführt.

Übersetzung des englischen Originaltextes: Christine Scholz, Klaus Zerres

Open Access. Dieser Artikel wird unter der Creative Commons Namensnennung 4.0 International Lizenz (http://creativecommons.org/licenses/by/4.0/deed. de) veröffentlicht, welche die Nutzung, Vervielfältigung, Bearbeitung, Verbreitung und Wiedergabe in jeglichem Medium und Format erlaubt, sofern Sie den/die ursprünglichen Autor(en) und die Quelle ordnungsgemäß nennen, einen Linkzur Creative Commons Lizenz beifügen und angeben, ob Änderungen vorgenommen wurden.

\section{Literatur}

1. Braude PR et al (1998) Non-disclosure preimplantation genetic diagnosis for Huntington's disease: Practical and ethical dilemmas. Prenat Diagn 18:1422-1426

2. Coonen E et al (1994) Optimal preparation of preimplantation embryo interphase nuclei for analysis by fluorescence in-situ hybridization. Hum Reprod 9:533-537

3. Dreesen JC et al (2000) Multiplex PCR of polymorphic markers flanking the CFTR gene; A general approach for preimplantation genetic diagnosis of cystic fibrosis. Mol Hum Reprod 6:391-396

4. Geraedts JP, De Wert GM (2009) Preimplantation genetic diagnosis. Clin Genet 76:315-325

5. Handyside AH et al (1990) Pregnancies from biopsied human preimplantation embryos sexed by Y-specific DNA amplification. Nature 344:768-770

6. Harper JC et al (2012) The ESHRE PGD Consortium: 10 years of data collection. Hum Reprod Update 18:234-247

7. Hundscheid RD et al (2001) Increased serum FSH in female fragile $X$ premutation carriers with either regular menstrual cycles or on oral contraceptives. Hum Reprod 16:457-462

8. Jasper MJ et al (2006) Singleton births after routine preimplantation genetic diagnosis using exclusion testing (D4S43 and D4S126) for Huntington's disease. Fertil Steril 85:597-602

9. Natesan SA et al (2014) Genome-wide karyomapping accurately identifies the inheritance of singlegene defects in human preimplantation embryos in vitro. Genet Med 16:838-845

10. Russell Z et al (2005) Thrombosis in a pregnant hemophilia A carrier after intrapartum recombinant factor VIII. Obstet Gynecol 105:875-876

11. Pepin Metal (2000) Clinical and genetic features of Ehlers-Danlos syndrome type IV, the vascular type. NEngl J Med 342:673-680

12. Scriven PN, Ogilvie CM (2007) Fluorescence in situ hybridization on single cells. (Sex determination and chromosome rearrangements). Methods $\mathrm{Mol}$ Med 132:19-23

13. Sermon K et al (1998) Preimplantation diagnosis for Huntington's disease (HD): Clinical application and analysis of the HD expansion in affected embryos. Prenat Diagn 18:1427-1436

14. Van Echten-Arends J et al (2013) Preimplantation genetic diagnosis for $X$; Aautosome translocations: Lessons from a case of misdiagnosis. Hum Reprod 28:3141-3145

15. Van Rij MC et al (2012) Preimplantation genetic diagnosis (PGD) for Huntington's disease: The experience of three European centres. Eur J Hum Genet 20:368-375 
Hier steht eine Anzeige.

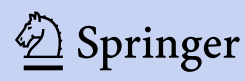

